\title{
Analysis of the jet pipe electro-hydraulic servo valve with finite element methods
}

\author{
Zhao Kaiyu $^{1}$, Wu Qingxun ${ }^{1}$, Zhang Lijian ${ }^{1}$, Yuan Zhaohui ${ }^{2}$ and Yang jiaqiang ${ }^{2}$ \\ ${ }^{1}$ Beijing Institute of Mechanical Equipment, 100854 Haiding district Beijing China \\ ${ }^{2}$ Northwestern Polytechnical University, 710129 Chang'an District of Xi'an, China
}

\begin{abstract}
The dynamic characteristics analysis about the jet pipe electro-hydraulic servo valve based on experience and mathematical derivation was difficult and not so precise. So we have analysed the armature feedback components, torque motor and jet pipe receiver in electrohydraulic servo valve by sophisticated finite element analysis tools respectively and have got physical meaning data on these parts. Then the data were fitted by Matlab and the mathematical relationships among them were calculated. We have done the dynamic multi-physical fields' Simulink co-simulation using above mathematical relationship, and have got the input-output relationship of the overall valve, the frequency response and step response. This work can show the actual working condition accurately. At the same time, we have considered the materials and the impact of the critical design dimensions in the finite element analysis process. It provides some new ideas to the overall design of jet pipe electro-hydraulic servo valve.
\end{abstract}

\section{Introduction}

Electro-hydraulic servo valve is used in many modern control systems. Due to the working oil pollution is a major factor that causes the servo valve does not working properly, the anti-pollution ability of the servo valve is very important. This servo valve driven by the pre-stage jet pipe nozzle, and the harm caused by the debris and impurity particles is no too bad, conversely, it's very easy to maintenance management, and its anti-pollution ability is very efficient.

The jet pipe electro-hydraulic servo valve which was driven by jet pre-stage has been applied in many prospects, so its characteristics has been put in-depth research. The jet pipe servo valve itself contains a magnetic, electrical, hydraulic multi-fields coupling analysis, every part is very complex, and the coupled fluids analysis cannot be carry out in any simple way. So the study of it has become more and more difficulty. Many of the existing products have a lot of problems, for example, the dead zone is too large, poor linearity, connection instability, poor dynamic characteristics etc. Especially when the valve constitutes a closed-loop system with steering gear, it is prone to instability. Further study of jet pipe electrohydraulic servo valve is necessary [1].

\section{The overall structure and principles of a jet pipe valve}

The total composition of jet pipe hydraulic servo valve can be divided into four parts: Moving iron permanent magnet torque motor, jet pipe amplifier, slide valve and feedback components. The coordinating relations among the various components were established through force feedback principle. The overall actions of the jet pipe electro-hydraulic hydraulic servo valve were described as below.

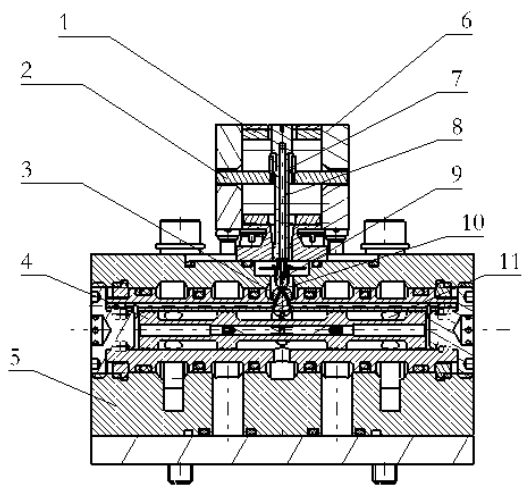

Figure 1. Front view of jet-tube hydraulic servo valve

In this figure, 1: Weak permeable material, 2: Armature and coil, 3: receiving hole, 4. Spool, 5: valve sleeve, 6: Strong permeable material, 7: The spring tube, 8: Jet pipe, 9: nozzle, 10: Overflow chamber 11:O-ring.

When we input a control current $i$ to the torque motor, the armature will produce a deflection angle $\theta$, so the jet pipe is deflected from the neutral position, the nozzle displacement was $x^{p}$. The fluid flow accepted by 
the two receiving holes of the nozzle below is different; the pressure differential would be $\Delta \mathrm{p}=p_{1}-p_{2}$ formed in the nozzle receiving chamber. The pressure difference drives the spool and feedback stick move to one end about $x_{v}$. When feedback stick continue deformation, the feedback force from stick and fluid pressure effects on ends of spool reaches dynamic equilibrium. In this case, the torque from feedback lever, spring tube, electromagnet torque motor, inlet pipe as well as the safety wire and leaf spring reach equilibrium.

\section{Feedback spring assembly stiffness calculation}

Feedback spring assembly was composed with the lever and connects pieces. The movement of the spool affecting the nozzle angle of deflection through feedback spring assembly. Use the Catia static analysis, put armature a rotating electromagnetic torque to analog actual circumstances, a direct force applied to the end of the feedback lever, the jet tube will be rotated a certain angle, the nozzle in response to a slight displacement. When put a Rotational torque on the armature, it is more obvious to cause the displacement of the jet pipe fuel injector end, the inlet pipe and twisted wire. So, fuel tube and twisted wire will produce a reaction torque on jet pipe. Furthermore, the lower part of the feedback spring tube assembly also have deformation accompanied the jet pipe nozzle [2].

If we give electromagnetic torque on the armature and force on the lower end of the feedback lever, we will get deflection displacement of the jet pipe. Record the relationship among them; we can analyze stiffness of the feedback spring assembly about the jet pipe. The various parts of the stress cloud as follows:
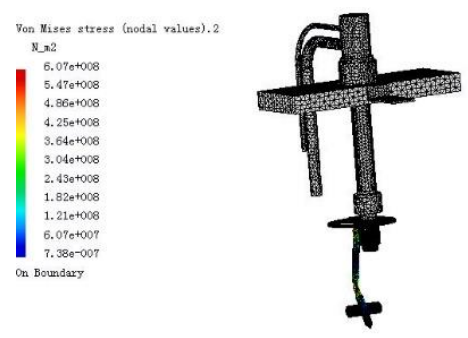

Figure 2. Armature feedback component stress cloud

Throughout the jet pipe, armature and feedback spring assembly, the force is mainly concentrated in the parts of armature and feedback spring where binding with the jet pipe. In addition, the parts of inlet pipe and twisted wire where connect with jet pipe were also the larger force area. As written in Eq.(1), $M_{1}$ indicates reaction torque of the leaf spring. $M_{2}$ indicates reaction torque of the spring tube. $M_{3}$ indicates reaction torque of the twisted wire. $M_{4}$ indicates reaction torque of the oil conduit. $M_{f}$ indicates reaction torque of the feedback stick.

$$
M_{0}=M_{1}+M_{2}+M_{3}+M_{4}+M_{f}
$$

It is not only related to the deflection angle of the jet pipe, but also the motion displacement of the spool. Then we take use of the Catia finite element simulation and get the relationship data.The mathematical relationships between them were fitted as follows:

$$
\begin{gathered}
M_{f}=27.28 \theta+311.85 x_{v}+3.53 \times 10^{-5} \\
f=10754 x_{v}+277.98 \theta+4.59 \times 10^{-5}
\end{gathered}
$$

\section{Torque motor characteristics analysis}

The torque motor in jet pipe electrohydraulic servo valve was an iron moving torque motor, which armature rotation direction is parallel to the working air gap flux density. This torque motor was constituted with permanent magnet, the upper guide magnet, the lower guide magnet, armature and the control coil. Permanent magnet magnetized the upper and lower guide magnet as the Arctic and the Antarctic. Two control coils trap around the armature. There will constitute four working air gap between the armature and the guide magnet. The magnetic permeability of the working air gap was showned respectively:

$$
\begin{gathered}
G_{1}=\frac{\mu_{0} A_{g}}{g-x} \\
G_{2}=\frac{\mu_{0} A_{g}}{g+x}
\end{gathered}
$$

Where $A_{g}$ is the area of the air gap, $g$ is the thickness of the air gap when armature is at the neutral position. $\mathrm{x}$ is the displacement of ends of the armature (pole face center) from the neutral position. We have plused the magnetic drop in the working air gap which were generated by fixed magnetic force and control magnetic potential. Then we will get the total magnetic pressure drop in working air gap:

$$
\begin{gathered}
U_{1}=\frac{B_{p} g}{\mu_{0}}+\frac{B_{c} g}{\mu_{0}} \\
U_{2}=\frac{B_{p} g}{\mu_{0}}-\frac{B_{c} g}{\mu_{0}}
\end{gathered}
$$

Where $B_{p}$ is the fixed magnetic flux density in the air gap when armature was at the neutral position. $B_{C}$ is equivalent to the control magnetic flux density when the armature in the neutral position. Now we can calculate the electromagnetic suction of each working air gap by the formula (4), (5), (6), (7) which were enumerated above. Then we can obtain the electromagnetic torque as follows[3]:

$$
M_{d}=\frac{20.4 \times 10^{-8} B_{p} A_{g} a N_{c}}{g} i_{c}+\frac{40.8 \times 10^{-8} B_{p}{ }^{2} A_{g} a^{2}}{\mu_{0} g} \theta
$$

Models and magnetic field distribution was shown in the following figure: 


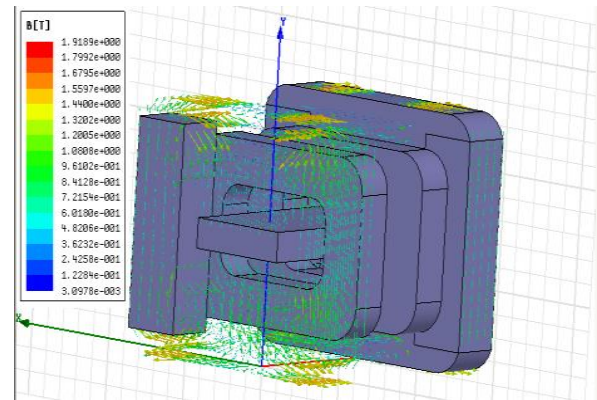

Figure 3. Magnetic field distribution of torque motor

The electromagnetic torque generated by the motor was ralated to the coil current and the armature deflection angle. Changing the deflection angle of the armature and the current respectively, we will obtain the relationship among them:

$$
M d=-0.002328+2.838 i c+16.79 \theta
$$

This is the relationship among electromagnetic torque, current and the armature declination obtained by the finite element analysis methods. It is very important for the analysis of whole servo valve system.

\section{Analysis of the flow field between the jet pipe and receiver}

We select the ansys workbench 13 as the tool of fluid simulation. The total process Include pre-processing (modeling, material properties, meshing, etc.), load, and solve (applying the boundary conditions, determine the solution method, etc.), post-processing (view and display)[4]. We meshed different parts of the model by tetrahedral and hexahedral use ANSYS ICEM CFD, and made 2 scale local grid refinements of the imports. The mesh size is 0.1 or 0.2 and the number of units is about 447,000. We use the SIMPLE method to solve the simulation. Fluid model use the k-epsilon two-equation model, When the nozzle deflection is $0.2 \mathrm{deg}$, convergence precision is about $10 \wedge-5$. The partial enlarged velocity vector diagram of nozzle - receiving hole was shown below:

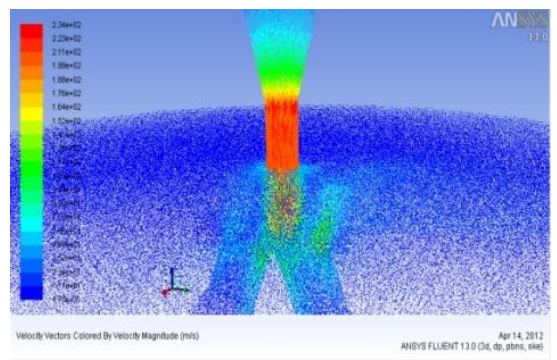

Figure 4. The velocity vector partial enlarged view of 0.2 degrees nozzle declination

We simulate the pressure difference at the two sides of spool by jet pipe CAD models in different declination. We consider the jet pipe is deflected in one direction only due to the spool movement and jet pipe deflection is symmetrical. The calculation results was shown in Fig.5:

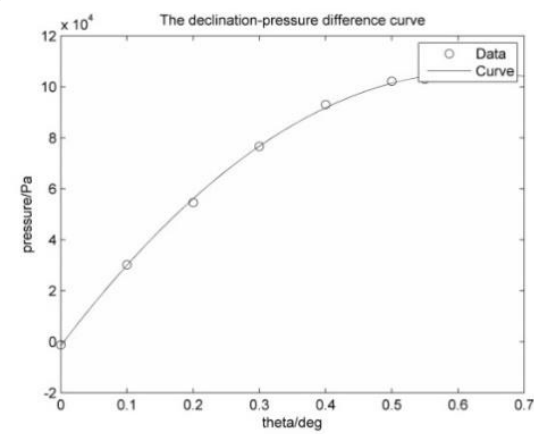

Figure 5. Pressure differential curve of different jet pipe declination

It is visible that the flow rate increases into a cavity and decreases into the other when the jet pipe is offset to one side. So the pressure difference increases gradually, this relationship can be approximated by a equation:

$$
\Delta p=-1.878 \times 10^{11} \theta^{2}+3.175 \times 10^{9} \theta+4.388 \times 10^{5}
$$

This is the relationship between the jet pipe declination and pressure differential at both ends of spool.

\section{Analysis of jet pipe electrohydraulic servo valve performance}

\section{1 mathematical models}

The stiffness of feedback lever and spring tube was confirmed by torque balance of armature assembly. The torque balance equation of armature assembly was [5]:

$$
\begin{aligned}
& K_{i} i_{c}(S)+K_{m} \theta(S)=J_{a} \ddot{\theta}+B_{a} \dot{\theta}+K_{a} \theta+r A_{p} p_{p} \\
& -r^{2} 8 \pi C_{p}^{2} p s x_{d} \theta+(r+b) K_{f}\left[(r+b) \theta+x_{v}\right]
\end{aligned}
$$

The left side of equation was electromagnetic torque of the torque motor, while the right was the anti-torque on the armature, the last anti-torque was came from the deformation of feedback lever. The pressure differential of spool ends was $\Delta \mathrm{p}=p_{1}-p_{2}$, one force $F_{f}$ applied to the spool came from the feedback lever, the other force $\Delta \mathrm{p}$ - Acame from fluid pressure differential of the spool ends. The kinetic equilibrium equation can be listed:

$$
\Delta P \cdot A-F_{f}=m \cdot \ddot{x}_{v}+\beta \cdot \dot{x}_{v}+F_{f f}\left(\dot{x}_{v}\right)
$$

We can get the finite element model of the whole valve through the analysis builded before.

\subsection{Simulation and Modeling}

The armature feedback components, torque motor and the jet pipe amplifier section has been simulated by the finite element software. We need to connect the various parts to build the overall model. The armature assembly damping is very important for the torque balance equation of torque motor. In addition, when we came to the force balance equation of spool parts, the valve internal loss along the tubule way should also be considered, as well as the steady-state fluid power. Given input current is sinusoidal, the amplitude is 0.043 and frequency is $200 \mathrm{~Hz}$. The relationship between the output displacement of spool and input signal was shown as below: 


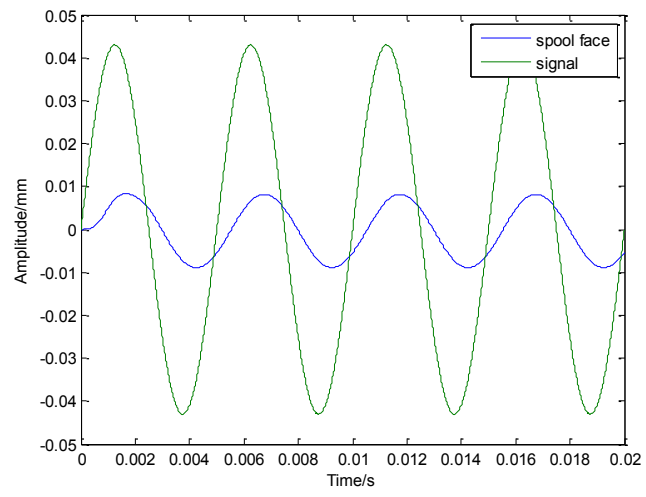

Figure 6. The relationship between Input signal and output displacement of spool

It can be seen that the spool displacement has a phase lag relative to the signal, but it can follow well. The frequency response characteristic of the whole system was shown as follows:

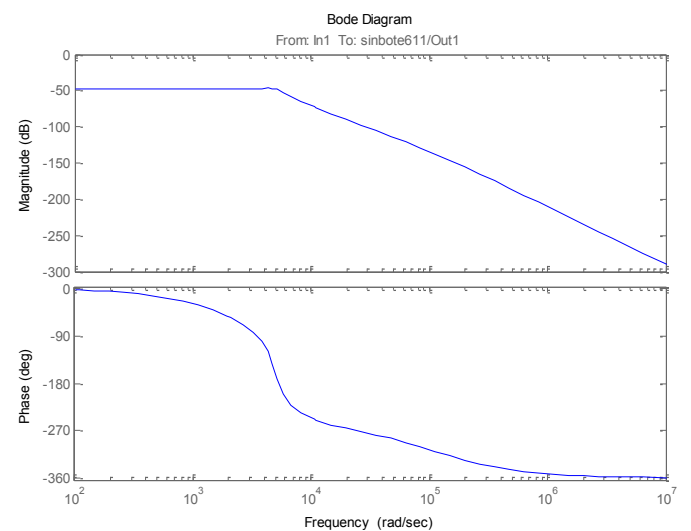

Figure 7. The frequency response curve of jet pipe electrohydraulic servo valve

The frequency response is the essential characteristics of the servo valve in the frequency domain, related to the geometrical structure, material, external working conditions and other factors, determined by the torque motors, slide valve and jet tube amplifier. Jet-tube hydraulic servovalve frequency responses have second-order characteristics related to the power sliding valve.

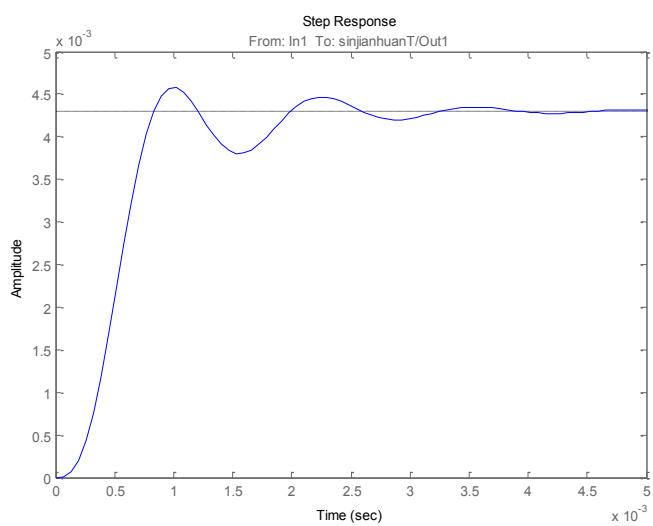

Figure 8. The step response curve of jet pipe electrohydraulic servo valve
Step response related to the oil viscosity, friction characteristics and dynamic characteristics of the spool primarily. As can be seen from the figure, the adjust time of servo valve is $0.005 \mathrm{~s}$, the overshoot is $12.5 \%$, which is obviously an ideal state, the adjusting time will be longer slightly in actual test.

\section{Conclusions}

We obtained the relationship of the key structures about jet pipe electrohydraulic servo valve by finite element simulation calculation, and summed up the dynamic characteristics of the jet pipe servo valve.

(1) We got the overall stiffness of armature feedback components by the method of finite element simulation, and obtained the relationship of the jet pipe deflection angle and electromagnetic torque in the case of certain feedback force.

(2) We got the relationship about electromagnetic torque, current and armature deflection angle by the finite element electromagnetic analysis.

(3) Analyze the relationship between the jet pipe declination and spool pressure at both ends through the ansys Fluent module.

(4) Analyze the overall dynamic characteristics of the valve with the mathematical simulation tools Matlab Simulink, We have got the relationship between input and output signal, the frequency response curve and the step response curve.

(5) We can got more reasonable and accurate results in simulation of the jet pipe valve through finite element analysis methods. We should take full advantage of the advanced simulation tools to obtain satisfactory results in the design process.

\section{References}

1. AV Mesropyan, RR Sharipov, Mathematical Modeling of Transient Processes in the Jet Pipe Servoactuator with a Dual-Mode Controller [J]. Procedia Engineering, (2016)

2. Xuan Hong Son Pham, Yao Bao Yin, Xi Zhang. Optimal Design for Torque Motor of Jet Pipe Electro-hydraulic Servo-valve Based on Dynamic Stiffness [C]. Intelligent Human-Machine Systems and Cybernetics (IHMSC), (2012)

3. M Jelali, A Kroll. Hydraulic servo-systems: modelling, identification and control [M].P. 62-68 (2012)

4. G.M. Ivanov, V.K Sveshnikov. Power efficient improving of digital hydraulic servo actuator Engineer Vestnik,7. P. 29-37. (2015)

5. AV. Mesropyan, RR. Sharipov. The simulation of complex digital control systems in the hydraulic servoactuater, RAS, Moscow. (2013) 